\title{
Evaluation of headache service quality indicators: pilot implementation in two specialist-care centres
}

\author{
Zaza Katsarava ${ }^{1,2^{*}}$, Raquel Gil Gouveia ${ }^{3}$, Rigmor Jensen ${ }^{4}$, Charly Gaul ${ }^{2,5}$, Sara Schramm ${ }^{6}$, Anja Schoppe ${ }^{2}$ \\ and Timothy J Steiner ${ }^{7,8}$
}

\begin{abstract}
Background: Evaluating quality of health care is increasingly recognized as an important contributor to the advancement of health-care delivery. We recently developed a set of quality indicators for headache care, intended to be applicable across countries, cultures and settings so that deficiencies in headache care worldwide might be recognized and rectified. These indicators themselves require evaluation and proof of fitness for purpose. This pilot study begins this process.

Methods: We tested the quality indicators in the tertiary headache centres of the University of Duisburg-Essen in Essen, Germany, and the Hospital da Luz in Lisbon, Portugal. Using seven previously-developed enquiry instruments, we interrogated health-care providers (HCPs), including doctors, nurses, psychologists and physiotherapists, as well as consecutive patients and their medical records.

Results: The questionnaires were easily understood by both HCPs and patients and were not unduly time-consuming The results from the two headache centres were comparable despite their differences in structure, staffing and language. These findings met the purpose of the study.

Diagnoses were made according to ICHD criteria and critically evaluated during follow-up. However, diagnostic diaries and instruments assessing burden and response to treatment were not always in place or routinely utilised. Triage systems adjusted waiting times to urgency of need. Treatment plans included pathways to other specialities. Patients felt welcomed, reassured and educated, and were mostly satisfied. Discussion points arose over inclusion of psychological therapies in treatment plans; over recording of outcomes; over indicators of efficiency and equitability (protocols to limit wastage of resources, systems to measure input costs and means of ensuring equal access to the services); and over protocols for reporting serious adverse events.
\end{abstract}

Conclusion: This pilot study to assess feasibility of the methods and acceptability of the instruments of headache service quality evaluation was successful. The project is ready to be taken into its next stages.

Keywords: Headache disorders; Headache care; Service quality evaluation; Global campaign against headache

\section{Background}

As a feature of health care, quality is obviously desirable. Evaluating quality of health care is increasingly recognized as a necessary link in the advancement of healthcare delivery. Yet it is not always clear what "quality" is in this context, and how it is achieved or whether it has been. With regard to headache care, quality has not-

\footnotetext{
* Correspondence: zaza.katsarava@uk-essen.de

'Department of Neurology, Evangelical Hospital Unna, Holbeinstrasse 10,

Unna 59423, Germany

2Department of Neurology, Western German Headache Centre, University of Duisburg-Essen, Essen, Germany

Full list of author information is available at the end of the article
}

until very recently-been defined; quality indicators for headache care that have been developed in the past, in the absence of any agreed meaning of "quality", are limited to diagnosis and treatment in specific health-care settings, or to specific types of headache.

To address this, so that deficiencies in headache care worldwide might be recognized and rectified, the Global Campaign against Headache $[1,2]$ in collaboration with the European Headache Federation (EHF) brought together a service quality evaluation (SQE) group of health-services researchers and headache specialists. Their first completed task, after a literature review [3], 
was to develop a definition of quality, which they conceived as residing in nine separate domains (see Table 1):

"Good quality headache care achieves accurate diagnosis and individualized management, has appropriate referral pathways, educates patients about their headaches and their management, is convenient and comfortable, satisfies patients, is efficient and equitable, assesses outcomes and is safe" [4].

Quality evaluation would require application of sets of quality indicators for each of these nine domains separately, and formulation of these was the group's second completed task [4]. They developed 30 in all, along with related assessment instruments, designed to be applicable across countries, cultures and settings.

Implementation requires the deployment of all of these, at various levels within health systems, most particularly in primary care, which is where most headache should be managed $[5,6]$. But prior to this, a number of evaluation studies are needed-in multiple settings, countries and cultures, to demonstrate acceptability of the instruments, with low barriers to usage, and fitness for purpose. Evaluation will require interrogation of healthcare providers (HCPs), including doctors, nurses, psychologists and physiotherapists, and of patients, which is not otherwise part of implementation.

Here we report the pilot evaluation study, undertaken in two specialist centres in Europe primarily to learn whether such an enquiry is feasible, and whether the questionnaires are easily understood by both HCPs and patients and not unduly time-consuming. The study continues the collaborative project (headache service quality evaluation) between EHF and Lifting The Burden

Table 1 The nine domains of quality in a headache service (4) Domain A: diagnostic accuracy, therefore asking whether diagnosis were made according to the IHS criteria, documented during the first visit and reviewed during he follow-ups and supported by the diagnostic diaries.

Domain B: issues of the individualized management including waiting time, use of diaries and instruments of headache related disability in treatment plans.

Domain C: availability and utilization of urgent and specialist referral pathways.

Domain D: patient's education and reassurance

Domain E: convenience, comfort and welcoming of the clinic

Domain F: patient's satisfaction

Domain G: equity and efficiency of the headache care including access to care, wastage of resources, rate of technical investigations and costs.

Domain $\mathrm{H}$ : outcome measures including clinical parameters but also measures of disability and quality of life.

Domain I: safety of care
(LTB) within the Global Campaign against Headache $[1,2]$, which is conducted by LTB in official relations with the World Health Organization.

\section{Methods}

The study was approved by the ethics committees of the University of Duisburg-Essen and Hospital da Luz. Informed consent was obtained from all study participants.

\section{Study settings and subjects}

The headache clinic of the University of Duisburg-Essen is a tertiary headache centre established within a major teaching hospital in Essen, Germany. Medical care is delivered as outpatient or day-clinic care by one senior physician, two residents, two psychologists and one physiotherapist supported by two nurses/secretaries. The centre is supported as necessary by other specialties within the hospital.

The headache clinic of the Hospital da Luz is a specialized centre within a private hospital in Lisbon, Portugal. Medical care includes a daily outpatient clinic, specialist support to the emergency department and inpatient care. One neurology consultant coordinates the centre, which works with physicians from multiple other specialities (physical and rehabilitation medicine, psychiatry, dentistry, gynaecology and obstetrics) and with other HCPs from the hospital, including psychologists, physiotherapists and nurses. The centre has one part-time secretary who coordinates care with the clinical secretaries of each speciality.

In each centre we interviewed the HCPs most involved in delivering the service, and studied consecutive patients and their records. Numbers of patients at each centre were determined by what were feasible.

\section{Instruments}

There were five instruments in total. Among these were three questionnaires: one each for doctors, other HCPs and patients. The last took the form of an exit questionnaire, which patients were asked to complete and return within 2 weeks. In addition, some items of information were extracted from the patients' records and from central service records.

Table 2 provides an overview of the methods and instruments used. The indicators studied (column one) are grouped in their domains A to I as defined by the SQE group [4]. Column three (Means of enquiry) indicates the method of enquiry: e.g., by questionnaire or chart review. Column two (Measure) explains how each was assessed (many as "yes"/"no", some quantitatively as continuous measures).

Questionnaires were translated from their English originals into German and Portuguese by three authors (ZK, CG and RG) according to Lifting The Burden's 
Table 2 Methods and outcomes of implementation of quality indicators in each centre

\begin{tabular}{|c|c|c|c|c|c|}
\hline \multirow{2}{*}{\multicolumn{2}{|c|}{ Indicator }} & \multirow[t]{2}{*}{ Measure } & \multirow[t]{2}{*}{ Means of enquiry } & \multicolumn{2}{|c|}{$\begin{array}{l}\text { Evaluators by centre } \\
\text { (percentages are of } \\
\text { positive responses) }\end{array}$} \\
\hline & & & & Essen & Lisbon \\
\hline \multicolumn{6}{|c|}{ Domain A. Accurate diagnosis is essential for optimal headache care } \\
\hline A1 & $\begin{array}{l}\text { Patients are asked about the temporal } \\
\text { profile of their headaches }\end{array}$ & $\begin{array}{l}\text { a) Duration of presenting complaint is } \\
\text { recorded in patient's record (yes/no) }\end{array}$ & patients' records & $99 \%$ & $100 \%$ \\
\hline \multirow[t]{2}{*}{ A2 } & $\begin{array}{l}\text { Diagnosis is according to current ICHD } \\
\text { criteria }\end{array}$ & $\begin{array}{l}\text { a) Diagnosis is recorded in patient's } \\
\text { record (yes/no) }\end{array}$ & patients' records & $100 \%$ & $100 \%$ \\
\hline & & $\begin{array}{l}\text { b) Diagnostic record uses ICHD terminology } \\
\text { (yes/no) }\end{array}$ & patients' records & $100 \%$ & $92 \%$ \\
\hline A3 & $\begin{array}{l}\text { A working diagnosis is made at the first } \\
\text { visit }\end{array}$ & $\begin{array}{l}\text { Working diagnosis at first visit is recorded } \\
\text { in patient's record (yes/no) }\end{array}$ & patients' records & $100 \%$ & $92 \%$ \\
\hline A4 & $\begin{array}{l}\text { A definitive diagnosis is made at first or } \\
\text { subsequent visit }\end{array}$ & $\begin{array}{l}\text { Definitive diagnosis is recorded in patient's } \\
\text { record or, if not, an appointment for review } \\
\text { has been given (yes/no) }\end{array}$ & patients' records & $98 \%$ & $92 \%$ \\
\hline A5 & $\begin{array}{l}\text { Diagnosis is reviewed during later } \\
\text { follow-up }\end{array}$ & $\begin{array}{l}\text { Diagnostic review during follow-up is } \\
\text { routinely undertaken (yes/no) }\end{array}$ & doctors' questionnaire & $100 \%$ & $100 \%$ \\
\hline A6 & $\begin{array}{l}\text { Diaries are used to support or confirm } \\
\text { diagnosis }\end{array}$ & $\begin{array}{l}\text { The service has a diagnostic diary } \\
\text { available, and doctors are aware of its } \\
\text { availability (yes/no) }\end{array}$ & doctors' questionnaire & $100 \%$ & $100 \%$ \\
\hline \multicolumn{6}{|c|}{ Domain B. Individualized management is essential for optimal headache care } \\
\hline \multirow[t]{2}{*}{ B1 } & $\begin{array}{l}\text { Waiting-list times for appointments are } \\
\text { related to urgency of need }\end{array}$ & $\begin{array}{l}\text { a) Waiting-list times are recorded in } \\
\text { database (yes/no) }\end{array}$ & patients' records & $0 \%$ & $0 \%$ \\
\hline & & $\begin{array}{l}\text { b) A formal triage system exists to expedite } \\
\text { appointments in cases of perceived } \\
\text { urgency (yes/no) }\end{array}$ & doctors' questionnaire & yes & yes \\
\hline \multirow[t]{5}{*}{ B2 } & $\begin{array}{l}\text { Sufficient time is allocated to each visit } \\
\text { for the purpose of good management }\end{array}$ & $\begin{array}{l}\text { a) Actual time (minutes) per visit } \\
\text { is recorded by patient in exit } \\
\text { questionnaire: } 1 \text { st visits }\end{array}$ & patients' questionnaire & $46 \pm 30$ & $25 \pm 7$ \\
\hline & & follow up visits & & $27 \pm 30$ & $24 \pm 9$ \\
\hline & & $\begin{array}{l}\text { b) Patient is satisfied }{ }^{a} \text { with actual time } \\
\text { (yes/not yes) }\end{array}$ & patients' questionnaire & $100 \%$ & $92 \%$ \\
\hline & & \multirow{2}{*}{$\begin{array}{l}\text { c) Health-care providers express overall } \\
\text { satisfaction (yes/no) }\end{array}$} & patients' questionnaire & $83 \%$ & $100 \%$ \\
\hline & & & $\begin{array}{l}\text { doctors' and other HCPs' } \\
\text { questionnaires }\end{array}$ & & \\
\hline B3 & $\begin{array}{l}\text { Patients are asked about the temporal } \\
\text { profile of their headaches }\end{array}$ & $\begin{array}{l}\text { Frequency (or days/month) of symptoms } \\
\text { is recorded in patient's record (yes/no) }\end{array}$ & patients' records & $100 \%$ & $100 \%$ \\
\hline B4 & $\begin{array}{l}\text { Treatment plans follow evidence-based } \\
\text { guidelines, reflecting diagnosis }\end{array}$ & $\begin{array}{l}\text { Prescribed drugs (names, doses and } \\
\text { quantities) are recorded in patient's record }\end{array}$ & patients' records & $100 \%$ & $96 \%$ \\
\hline \multirow[t]{2}{*}{ B5 } & $\begin{array}{l}\text { Treatment plans include psychological } \\
\text { approaches to therapy when appropriate }\end{array}$ & $\begin{array}{l}\text { a) Access route to psychological } \\
\text { therapies exists (yes/no) }\end{array}$ & doctors' questionnaire & yes & yes \\
\hline & & b) Utilisation is recorded in patient's record & patients' records & $100 \%$ & $32 \%$ \\
\hline \multirow[t]{3}{*}{ B6 } & $\begin{array}{l}\text { Treatment plans reflect disability } \\
\text { assessment }\end{array}$ & $\begin{array}{l}\text { a) An instrument for disability assessment } \\
\text { is available (ves/no) and is appropriate }\end{array}$ & doctors' questionnaire & yes & yes \\
\hline & & in the setting (yes/no) & & yes & yes \\
\hline & & $\begin{array}{l}\text { b) Disability is recorded in patient's record } \\
\text { (yes/no) }\end{array}$ & patients' records & $0 \%$ & $100 \%$ \\
\hline \multirow[t]{2}{*}{ B7 } & $\begin{array}{l}\text { Patients are followed up to ascertain } \\
\text { optimal outcome }\end{array}$ & $\begin{array}{l}\text { a) Follow-up appointment dates appear in } \\
\text { central service records }\end{array}$ & central service records & $36 \%$ & $32 \%$ \\
\hline & & $\begin{array}{l}\text { b) A follow-up diary and/or calendar is } \\
\text { available (yes/no) }\end{array}$ & doctors' questionnaire & yes & yes \\
\hline
\end{tabular}


Table 2 Methods and outcomes of implementation of quality indicators in each centre (Continued)

Domain C. Appropriate referral pathways are essential for optimal headache care

C1 Referral pathway is available from $\quad$ A usable pathway exists (yes/no) primary to specialist care

C2 Urgent referral pathway is available A usable pathway exists (yes/no) when necessary

doctors' questionnaire yes yes
doctors' questionnaire yes yes

Domain D. Education of patients about their headaches and their management is essential for optimal headache care

D1 Patients are given the information they need to understand their headache and its management

D2 Patients are given appropriate reassurance

\section{Patient is satisfied ${ }^{a}$ with information given (yes/not yes) \\ Patient is satisfied ${ }^{\mathrm{a}}$ with reassurance given (yes/not yes)}

Domain E. Convenience and comfort are part of optimal headache care
E1 The service environment is clean and comfortable

E2 The service is welcoming

E3 Waiting times in the clinic are acceptable a) Patient is satisfied ${ }^{a}$ with cleanliness and comfort (yes/not yes)

b) Health-care providers are satisfied with cleanliness and comfort (yes/no)

Patient is satisfied ${ }^{a}$ with welcome (yes/not yes)

a) Waiting time (minutes) is recorded by patient in exit questionnaire

b) Patient is satisfied ${ }^{a}$ with waiting time (yes/not yes)

c) Health-care providers are satisfied with waiting times (yes/no) patients' questionnaire

patients' questionnaire

$100 \%$

patients' questionnaire

doctors' and other

HCPs' questionnaires

patients' questionnaire

$100 \%$

$94 \%$

patients' questionnaire

$20 \pm 18$

$23 \pm 23$

patients' questionnaire

$88 \%$

doctors' and other

$100 \%$

$60 \%$ HCPs' questionnaires

patients' questionnaire management (yes/not yes) management

\section{equitable}

G1 Procedures are followed to ensure

A protocol to limit wastage exists (yes/no)

doctors' questionnaire

no

no

doctors' questionnaire yes yes

G2 Costs of the service are measured as part of a cost-effectiveness policy

A record of input costs exists (yes/no)

A policy to ensure equal access exists (yes/no)

doctors' questionnaire

no

no services for all who need it

a) An outcome measure (HURT or similar) is available (yes/no)

b) Outcomes according to this measure are recorded in patient's record (yes/no/not applicable)

H2 Outcome measures are based on self-reported disability burden

a) An outcome measure (HALT or similar) is available (yes/no)

b) Outcomes according to this measure are recorded in patient's record (yes/no/not applicable)

H3 Outcome measures are based on self-reported quality of life

a) An outcome measure (WHOQoL or similar) is available (yes/no)

b) Outcomes according to this measure are recorded in patient's record (yes/no/not applicable)

Domain I. Optimal headache care is safe

11 Patients are not over-treated ${ }^{\text {b }}$

Prescribed drugs (names, doses and quantities) are recorded in patient's record (yes/no/not applicable) doctors' questionnaire yes yes

patients' records

doctors' questionnaire

no

yes

patients' records

na

$68 \%$

doctors' questionnaire

no

no

patients' records

na

na
$100 \%$

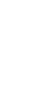


Table 2 Methods and outcomes of implementation of quality indicators in each centre (Continued)

\begin{tabular}{|c|c|c|c|c|c|}
\hline \multirow[t]{2}{*}{12} & \multirow{2}{*}{$\begin{array}{l}\text { Systems are in place to be aware of } \\
\text { serious adverse events }{ }^{c}\end{array}$} & a) Serious adverse events are recorded & $\begin{array}{l}\text { patients' records, central } \\
\text { service records }\end{array}$ & none & none \\
\hline & & $\begin{array}{l}\text { b) A protocol exists for reporting } \\
\text { serious adverse events (yes/no) }\end{array}$ & doctors' questionnaire & no & yes \\
\hline \multicolumn{6}{|c|}{$\begin{array}{l}\text { HCP Health-care provider, na not applicable, ICHD International Classification of Headache Disorders, HURT Headache Under-Response to Treatment questionnaire } \\
\text { [8], HALT Headache-Attributed Lost Time questionnaire [10] } \\
\text { a Patient's satisfaction was elicited either from the options "yes" / "no", or as the middle option of "too much" / "about right" / "too little", or as "very good" or } \\
\text { "good" on a Likert scale extending through "adequate", "poor" and "very poor" } \\
\text { "Over-treatmentmay mean excessive use of drugs likely to induce MOH, overdosage with potentially harmful drugs such as ergotamine or steroids, use of } \\
\text { prophylactics for infrequent headache, use of prophylactics for the wrong diagnosis, or use of non-evidence-based treatments that are unlikely to be effective } \\
\text { land may jeopardize safety } \\
\text { "Serious adverse events are those that cause death, are life-threatening, terminate or put at risk a pregnancy, or cause hospitalization, prolonged illness, disabilit) } \\
\text { and/or malignancy }\end{array}$} \\
\hline
\end{tabular}

translation protocol for hybrid documents [7], using forward and backward translation, with colleagues and patients not involved in the study providing review and assistance.

\section{Data collection}

HCPs and patients completed questionnaires anonymously. Data from patients' records were extracted by a medical student (AS) in Essen and by a clinical research assistant in Lisbon. Information from central service records was supplied by the HCPs.

\section{Analysis}

Demographic and clinical data are provided as numerical values, percentages or mean values with standard deviations (SDs). No statistical tests were performed.

\section{Results}

In Essen we interviewed six HCPs: one senior physician, two residents, two nurses and one psychologist. We interviewed and reviewed the records of 89 patients, of whom 22 were male, 67 female, mean age $42.5 \pm 12.7$ years. Most (61) had migraine, 53 episodic and eight chronic; 13 had tension-type headache (TTH), 9 episodic and four chronic; 18 had medication-overuse headache $(\mathrm{MOH})$, one had cluster headache $(\mathrm{CH})$ and one had trigeminal neuralgia (TN). Five patients were given two diagnoses.

In Lisbon we interviewed $10 \mathrm{HCPs}$ : one neurologist, one dental surgeon, two psychiatrists, two physiotherapists and four gynaecologists. We interviewed and reviewed the records of 50 patients, five male and $45 \mathrm{fe}$ male, mean age $37.3 \pm 11.2$ years. Again, most patients (40) had migraine, seven had TTH, four had $\mathrm{MOH}$ and one each had new daily persistent headache, TN and orgasmic cephalalgia. Four patients were given two diagnoses.

On a general level, questionnaires proved easy to apply, were readily understood and accepted by both HCPs and patients, and not unduly time consuming. None of the specific enquiries caused or led to difficulties. Evaluation of each clinic according to the quality indicators is shown in Table 2. Despite the two different languages, German and Portuguese, the different settings and the very different structures of the two centres, the findings were very comparable. We summarise them by domain.

\section{Domain A: accurate diagnosis}

Diagnoses were made according to current ICHD criteria at both centres, documented after the first visit and reviewed after follow-up with the support of diagnostic diaries. Percentages of positive responses were $\geq 98 \%$ in Essen and $\geq 92 \%$ in Lisbon (Table 2).

\section{Domain B: individualized management}

A triage system to identify urgent cases existed in both clinics, but waiting-list times until first appointment were not documented. Mean time allocated to patients' visits (according to patients' reports) was 46 min for the first visit in Essen (with wide variation), but half that (and with much less variation) in Lisbon. Patient satisfaction (Essen 100 \%, Lisbon $92 \%$ ) did not appear to be greatly influenced by this (Table 2). Treatment plans followed international guidelines. Lisbon made less use of psychological treatment. Disability measures existed in both clinics; disability was well documented in Lisbon (100\%), but not at all in Essen. In both clinics, only one third of patients were routinely followed to ascertain optimal outcomes (Table 2).

\section{Domain C: appropriate referral pathways}

Both clinics were well established with referral pathways for urgent and specialist consultations (Table 2).

\section{Domain D: education and reassurance of patients}

The great majority of patients expressed satisfaction (Essen $\geq 99 \%$; Lisbon $\geq 92 \%$ ) on both counts (Table 2).

\section{Domain E: convenience and comfort}

More patients (94-98 \%) than HCPs (60-67\%) found the service environment clean and comfortable; most patients felt welcomed (Essen 100 \%, Lisbon 94\%). Waiting times in the clinic varied quite widely around means of 20-23 min, and were unsatisfactory for a 
sizeable minority of patients (Essen $12 \%$, Lisbon $22 \%$ ) and $40 \%$ of HCPs in Lisbon (Table 2).

\section{Domain F: patient satisfaction}

Overall satisfaction with their management was expressed by most patients (Essen $96 \%$, Lisbon $74 \%$ ) (Table 2).

\section{Domain G: efficiency and equitability}

Neither clinic had a protocol to avoid wastage of resources. Running costs were calculated, but the information was available to senior management only. Neither clinic was able to offer equal access to headache services for all who might need it.

\section{Domain $\mathrm{H}$ : outcome assessment}

Outcome measures were available in both clinics (in Essen only for symptom burden, in Lisbon also for disability), but they were used only in Lisbon. Neither clinic evaluated quality of life.

\section{Domain I: safety}

The service was safe in Lisbon, and probably also in Essen. Prescribed drugs were always well documented in both centres. In both, also, there were no recorded serious adverse events (SAEs), but the pilot identified absence in Essen of any formal protocol to ensure that SAEs were reported (Table 2).

\section{Discussion}

This was a pilot study to assess feasibility of the methods and acceptability of the instruments before committing resources to a large multicentre implementation study, which-still in the evaluation phase of the project-must be done next. The pilot was successful in this purpose: the questionnaires were found easy to apply and were readily understood and acceptable, and, importantly, the whole process proved not unduly time consuming. All this was so in both German and Portuguese languages, and in two centres with very different structures-one in a university teaching hospital and one in a private hospital.

Also in these two very different settings, findings with regard to quality were quite comparable. We can summarise these findings, while emphasising that here we were testing the concepts behind the quality evaluation project, not the centres, and we were certainly not comparing the centres. Triage systems were in place to adjust waiting times to first appointment to urgency of need. Waiting times to be seen were generally considered acceptable and most patients felt welcomed, reassured and satisfied. Diagnoses were made according to current ICHD criteria, and were critically evaluated during follow-up visits. Treatment plans included psychological therapies (variably utilised) and pathways to other specialities. Outcomes were assessed on symptom measures (such as headache intensity and frequency) and on disability burden in Lisbon. However, instruments to assess outcomes were not always in place, or not routinely utilised.

Both services would be considered to have failed on indicators of efficiency and equitability. Protocols for limiting wastage of resources did not exist; input costs were measured, but the personnel utilising resources were not informed of them; equal access to the services was not ensured.

The services appeared safe, but we say more about this below.

There are two lines of thought worth further discussion. One is to look at these findings and, where problems appeared, question whether these truly reflected issues of quality. The other is to identify the next steps in evaluation of the quality indicators.

Pursuing the first of these, we see no issues arising in domain $\mathrm{A}$. In domain $\mathrm{B}$, the centres differed markedly in their utilisation of psychological management approaches (100 \% at Essen, $32 \%$ at Lisbon), although both had access. They differed even more in recording disability in patients' notes in order that management plans might reflect this (0\% at Essen, $100 \%$ at Lisbon). We will come back to whether or not these are issues of quality. Both centres showed evidence of routinely following-up only one third of patients. In primary care, the expectation would be close to $100 \%$, whereas specialist-care practice entirely appropriately sends many patients back to primary care, with detailed advice for follow-up. Domains $\mathrm{C}$ and $\mathrm{D}$ raised no issues, interestingly in the latter case since it recorded patient satisfaction, which is notoriously fickle. That fickleness may be evident in domain F: Lisbon may be disappointed with an overall patient satisfaction rating of $74 \%$ in the face of much higher scores elsewhere (although only $78 \%$ for waiting times-domain E). Do patients really attach so much more importance to waiting time than to time spent with the doctor (92\% satisfied), information received ( $92 \%$ ) and reassurance ( $94 \%$ ), cleanliness and comfort (94\%) and a warm welcome (94\%)? When developing these indicators, the SQE group found it impossible to exclude patient satisfaction [4], but it can be difficult to understand what are its determinants. In fact, waiting times were clearly a problem at Lisbon, since the HCPs expressed even less satisfaction with them (60\%), but only to a limited extent are they within the control of clinicians. These quality indicators are equally for service managers, who have responsibility for resource allocation and for protocols avoiding wastage of resources, and who, perhaps, can do something to promote equitable access.

The SQE group did not incorporate clinical outcomes themselves into the quality indicators because they 
found no objective basis for stipulating what outcomes should be considered optimal in individual cases in particular settings [4]. Instead they called for evidence that outcomes were recorded against recognised outcome measures. Neither centre apparently attached great importance to this: Lisbon made some use of recognised outcome measures in two thirds (68\%) of cases, Essen not in any. Both centres did, nonetheless, record outcomes in terms of symptoms-in particular, headache frequency-in patients' records. The quality issues related to this are threefold. First, accurate recording of outcomes serves patient follow-up and guides achievement of best outcomes. Second, it is part of good recordkeeping, failure of which would be clear evidence of a quality deficiency. However, recognised outcome measures are not needed for either of these purposes: any system that has meaning to its users may be sufficient, including simple but careful recordings of symptoms in patients' notes. But the third is this: how does a service know what its outcomes are; how can it assess itself against benchmarks; how can it improve (or even know whether it needs to improve)? This purpose is quite different, and requires that outcomes are formally recorded, in a standard manner, and it is for this purpose that recognised outcome measures are mandatory.

Since no outcome measures are yet universally accepted, it is part of the service quality evaluation project to suggest those that might be used and include them in the evaluations. The HURT questionnaire was developed as an outcome measure specifically for the purpose of achieving best outcomes [8]. While it was intended primarily for non-specialists, it has much broader utility [9], reflected in the fact that both centres had it available. Lisbon made use of it for the majority of their patients. For disability burden, the HALT questionnaire [10], like MIDAS from which it is derived [11], records lost productive timewhich correlates strongly with disability. The two instruments have been widely validated, and HALT is available in multiple European translations. As for quality of life, while WHOQoL-8 [12] was suggested, there is no evidence that this is useful as an outcome measure in headache management. Neither is there good support for any alternative. Future studies may conclude that this indicator should not be retained.

Finally, on the matter of safety, the issue again is one of record-keeping. It may be that a protocol for reporting SAEs at Essen had not been required because none had occurred, but managers should have recognised the need to have one in place. It is not a minor point. Essen would have been considered to have failed on this indicator: it could not be known for certain whether there had been no SAEs at Essen or whether they were merely not recorded.
The second line of thought is to consider the next steps in developing the methodology and instrumentation of headache service quality evaluation. Following this pilot, the quality indicators will be implemented in many centres in a Europe-wide study supported by the European Headache Federation and Lifting The Burden, still in specialist care. The protocol will be the same, with the seven instruments used to evaluate performance. This extended study will serve two purposes. First it will confirm (or not) that the indicators are fit for purpose, with or without some degree of refinement. Second, by establishing what is majority practice, it will guide the setting of benchmarks against which quality may be judged. Uncertainties, such as the extent to which psychological approaches should be used in management, and whether and to what extent management plans should be based on disability assessments, on which Essen and Lisbon clearly differed, may not belong in indicators of quality. We shall look for consensus in this study. There will be both qualitative and quantitative benchmarks to be set. The danger, of course, is to assume that majority practice correctly sets the benchmark; but that is a matter for these future studies.

The stage after that will be to take the process into non-specialist care-primary care in particular. It is envisaged that the finally-agreed quality indicators should not themselves be varied when taken into primary care, but the benchmarks may be different.

\section{Conclusion}

This pilot study to assess feasibility of the methods and acceptability of the instruments of headache service quality evaluation was successful. The project is ready to be taken into its next stages.

\section{Abbreviations \\ $\mathrm{CH}$ : Cluster headache; EHF: European Headache Federation: \\ ICHD: International classification of headache disorders; HCP: Health-care practitioner; LTB: Lifting The Burden; $\mathrm{MOH}$ : Medication-overuse headache; SAE: Serious adverse event; SD: Standard deviation; SQE: Service quality evaluation; TTH: Tension-type headache; TN: Trigeminal neuralgia.}

\section{Competing interests}

ZK and RJ are directors and trustees of the European Headache Federation. ZK, RJ and TJS are directors and trustees of the Lifting The Burden.

No author has other conflicts of interest relevant to the subject matter of this manuscript.

\section{Authors' contributions}

ZK and TJS conceived the study and developed the protocol with input from RGG and RJ. ZK, SS, RGG, RJ, CG and AS collected and analysed the data. ZK and TJS drafted the manuscript. All authors were involved in critical review of the first and subsequent drafts, and approved the final version.

\section{Acknowledgements}

This study was supported by the European Headache Federation and by Lifting The Burden, a UK-registered non-governmental organisation conducting the Global Campaign against Headache in official relations with the World Health Organization. 


\section{Author details}

'Department of Neurology, Evangelical Hospital Unna, Holbeinstrasse 10, Unna 59423, Germany. ${ }^{2}$ Department of Neurology, Western German Headache Centre, University of Duisburg-Essen, Essen, Germany. ${ }^{3}$ Hospital da Luz Headache Centre, Neurology Department, Hospital da Luz, Lisbon, Portugal. ${ }^{4}$ Danish Headache Centre, Department of Neurology, University of Copenhagen, Glostrup Hospital, Copenhagen, Denmark. ${ }^{5}$ Migraine and Headache Clinic, Königstein, Germany. ${ }^{6}$ Institute of Epidemiology, Biometry and Statistics, University of Duisburg-Essen, Essen, Germany. ${ }^{7}$ Department of Neuroscience, Norwegian University of Science and Technology, Trondheim, Norway. ${ }^{8}$ Division of Brain Sciences, Imperial College London, London, UK.

Received: 18 March 2015 Accepted: 1 June 2015

Published online: 09 June 2015

\section{References}

1. Steiner TJ (2004) Lifting the burden: The global campaign against headache. Lancet Neurol 3:204-205

2. Steiner TJ (2005) Lifting The Burden: The global campaign to reduce the burden of headache worldwide. J Headache Pain 6:373-377

3. Peters M, Perera S, Loder E, Jenkinson C, Gil Gouveia R, Jensen R, Katsarava Z, Steiner TJ (2012) Quality in the provision of headache care. 1: systematic review of the literature and commentary. J Headache Pain 13:437-447

4. Peters $\mathrm{M}$, Jenkinson C, Perera S, Loder E, Jensen R, Katsarava Z, Gil Gouveia R, Broner S, Steiner T (2012) Quality in the provision of headache care. 2: defining quality and its indicators. J Headache Pain 13:449-457

5. Steiner TJ, Paemeleire K, Jensen R, Valade D, Savi L, Lainez MJA, Diener H-C, Martelletti $P$, Couturier EGM, on behalf of the European Headache Federation and Lifting The Burden: The Global Campaign to Reduce the Burden of Headache Worldwide (2007) European principles of management of common headache disorders in primary care. J Headache Pain 8(suppl 1):S3-S21

6. World Health Organization, Lifting The Burden (2011) Atlas of headache disorders and resources in the world 2011. WHO, Geneva

7. Peters M, Bertolote JM, Houchin C, Kandoura T, Steiner TJ (2007) Translation protocol for hybrid documents. J Headache Pain 8(suppl 1):S45-S47

8. Buse DC, Sollars CM, Steiner TJ, Jensen RH, Al Jumah MA, Lipton RB (2012) Why HURT? A review of clinical instruments for headache management. Curr Pain Headache Rep 16:237-254

9. Westergaard MLS, Steiner TJ, MacGregor EA, Antonaci F, Tassorelli C, Buse DC, Lipton RB, Jensen RH (2013) The Headache Under-Response to Treatment (HURT) questionnaire: assessment of utility in headache specialist care. Cephalalgia 33:245-255

10. Steiner TJ (2007) The HALT and HART indices. J Headache Pain 8 (suppl 1):S22-S25

11. Stewart WF, Lipton RB, Kolodner KB, Sawyer J, Lee C, Liberman JN (2000) Validity of the Migraine Disability Assessment (MIDAS) score in comparison to a diary-based measure in a population sample of migraine sufferers. Pain 88:41-52

12. Schmidt S, Mühlan H, Power M (2005) The EUROHIS-QOL 8-item index psychometric results of a cross-cultural field study. Eur J Public Health $16: 420-428$

\section{Submit your manuscript to a SpringerOpen ${ }^{\odot}$ journal and benefit from:}

- Convenient online submission

- Rigorous peer review

- Immediate publication on acceptance

- Open access: articles freely available online

- High visibility within the field

- Retaining the copyright to your article 